engage in compensated sex $(10 \% \mathrm{MU}$ vs. $4 \% \mathrm{FC}, \mathrm{p}<0.05)$, not use condoms during their last sexual encounter (64\% MU vs. $45 \% \mathrm{FC}$, $\mathrm{p}<0.05)$, and be a first-time HIV tester (50\% MU vs. $41 \% \mathrm{FC}$, $\mathrm{p}<0.05$; see Table 1).

MU HIV prevalence was $5 \%$ (vs. $17 \%$ FC, p < 0.05). Among firsttime testers, HIV prevalence in both MSM and TW was not significantly different between MU and FC attendees (MSM: 13\% MU vs. $19 \%$ FC $p=0.14$; TW: $41 \%$ MU vs. $50 \%$ FC $p=0.71$ ).

Conclusion MU testing reached large numbers of high-risk (TW/ MSM) and potentially bridging (MSMW) populations engaged in unsafe sexual behaviours. MU HIV prevalence for MSM/TW firsttime testers was similar to that of the FC, making MU outreach a worthy complement to FC testing. Further investigation into whether MU attendees would not otherwise access HIV testing is warranted to determine the impact of MU testing.

Abstract P5.033 Table 1 Proportion of first-time HIV testers in MU vs. FC attendees stratified by sexual identity

\begin{tabular}{llll}
\hline & $\begin{array}{l}\text { MU } \\
(\mathbf{n = 3 , 4 9 6 )}\end{array}$ & $\begin{array}{l}\text { FC } \\
(\mathbf{n = 1 , 8 5 4 )}\end{array}$ & p value \\
\hline MSM & $164 / 590(28 \%)$ & $366 / 1,031(36 \%)$ & $<0.05$ \\
TW & $42 / 282(15 \%)$ & $4 / 50(8 \%)$ & .19 \\
MSMW & $590 / 942(63 \%)$ & $88 / 154(57 \%)$ & .20 \\
Total & $1,764(50 \%)$ & $765(41 \%)$ & $<0.05$ \\
\hline
\end{tabular}

\section{P5.034 BRITISH CO-OPERATIVE CLINICAL GROUP (BCCG) PROJECT - HOW OFTEN ARE MEN WHO HAVE SEX WITH MEN (MSM) ADVISED TO ATTEND FOR REPEAT SEXUAL HEALTH SCREENING IN THE UK?}

doi:10.1136/sextrans-2013-051184.1079

${ }^{1} E$ Clarke, ${ }^{2} E$ Shone, ${ }^{2} \mathrm{C}$ Broad, ${ }^{3} \mathrm{~A}$ Baker, ${ }^{1,2} \mathbf{R}$ Patel, ${ }^{1} \mathrm{D}$ Rowen. ${ }^{1}$ Royal South Hants Hospital, Southampton, UK; ${ }^{2}$ Faculty of Medicine, University of Southampton, Southampton, UK; ${ }^{3}$ Faculty of Medicine, university of Southampton, Southampton, UK

Background Within the UK rates of HIV, STIs, and sexual risk taking behaviours are increasing in MSM. 24\% of new UK HIV diagnoses in MSM are recently acquired. Increased frequency of sexual health screening could reduce transmission of STIs, and this policy is advocated in Australia and America. UK guidelines are vague, advising increased screening for MSM with risk taking behaviours but without specifying frequency or behaviours. Aim: to assess what advice UK clinics give MSM about how frequently they should receive sexual health screening, and how this varies with risk taking behaviours.

Methods BCCG members representing UK level 3 sexual health clinics were asked to complete an online survey assessing how often they would advise MSM with different patterns of risk taking behaviours to attend for screening.

Results 53 clinics responded. $53 \%$ had local guidance on screening frequency, with $89 \%$ based on national guidelines. 94\% BCCG members and $59 \%$ local guidelines identified behaviours requiring increased screening frequency. The majority (53-66\%) advised 3 monthly screening for MSM with over 10 partners in the last 6 months, attending sex on premises venues, using recreational drugs during sex, using the Internet to find partners, participating in group sex, or being HIV positive and sexually active. $32 \%$ and $40 \%$ respectively recommended 6 monthly screening for sexually active MSM and for those who had unprotected anal sex in the last 12 months.

Discussion Whilst the majority of clinics surveyed would recommend 3-6 monthly screening for MSM with risk taking behaviours, this varies with many clinics providing no specific advice on screening frequency. Clear UK guidance is needed to respond to the HIV epidemic in MSM by promoting early diagnosis of incident infection, and to ensure that efforts to contain costs through service contraction do not impinge on access or screening for this important group.

\section{P5.035 WITHDRAWN BY AUTHOR}

\section{P5.036 THE FIRST CLUSTER RANDOMISED TRIAL OF A MOLECULAR CHLAMYDIA AND GONORRHOEA POINT-OF- CARE ASSAY}

doi:10.1136/sextrans-2013-051184.1080

1,2L Natoli, ${ }^{2,3} \mathrm{~J}$ Ward, ${ }^{2,4} \mathrm{~B}$ Hengel, ${ }^{2} \mathrm{~L}$ M Causer, ${ }^{2} \mathrm{~S}$ Badman, ${ }^{2,5} \mathrm{~A}$ Tangey, ${ }^{6,7} \mathrm{~S} N$ Tabrizi, ${ }^{8} \mathrm{D}$ Whiley, ${ }^{2} \mathrm{~B}$ Donovan, ${ }^{9,10} \mathrm{C}$ Fairley. ${ }^{1}$ The Burnet Institute, Melbourne, Australia; ${ }^{2}$ The Kirby Institute, University of New South UK, Sydney, Australia; ${ }^{3} B a k e r$ IDI, Central Australia, Alice Springs, Australia; ${ }^{4}$ Apunipima Health Council, Cairns, Australia; ${ }^{5}$ Ngaanyatjarra Health Service, Warburton, Australia; ${ }^{6}$ Department of Microbiology and Infectious Diseases, The Royal Women's Hospital, Parkville, Australia; ${ }^{2}$ 2) Department of Obstetrics and Gynaecology, University of Melbourne, The Royal Women's Hospital and Murdoch Childrens Research Institute, Parkville, Australia; ${ }^{8}$ Oueensland Paediatric Infectious Diseases Laboratory/Queensland Children's Medical Research Institute, Royal Children's Hospital, Herston, Australia; ${ }^{9}$ Melbourne School of Population Health University of Melbourne, Carlton, Australia; ${ }^{10}$ Melbourne Sexual Health Centre, Carlton, Australia

Introduction In many settings, control of sexually transmissible infections (STIs) is compromised by lack of laboratory infrastructure, physical distance from laboratories and loss to follow up of patients. Point-of-care (POC) tests have the potential to provide timely diagnosis, treatment and partner notification, and in turn reduce infection rates. In April 2013, we will implement the first cluster randomised trial of chlamydia and gonorrhoea POC testing in remote Aboriginal communities where STIs are endemic.

Methods The study, called TTANGO (test, treat and go), will measure the effectiveness, cost-effectiveness and cultural and operational acceptability of POC testing for chlamydia and gonorrhoea infections. The study design is a crossover, cluster, randomised controlled trial involving 12 health services in remote Aboriginal communities in Australia. The primary outcome is the percentage of people with persistent chlamydia and gonorrhoea positive tests. The trial runs for 2 years and is a partnership between research, government and community organisations.

Results TTANGO is reaching the conclusion of the preparation phase and has achieved significant steps, including the engagement of remote health services and communities, a comprehensive laboratory and field evaluation to select the ideal assay, development of the first formal training package for chlamydia and gonorrhoea POC testing, and a quality assurance programme. The GeneXpert ${ }^{\circledast} \mathrm{CT} /$ NG molecular POC assay was selected for the trial as it showed very high sensitivity and specificity compared to other assays, was easy to use and results were available in approximately 90 minutes. This paper will discuss progress in preparing and implementing TTANGO, the methodology and evaluation.

Conclusion The results of this RCT will provide crucial information to guide sexual health clinical practise in remote Aboriginal communities and other settings internationally. Mathematical modelling and health economic analyses will be used to make the case for large scale implementation of this technology.

\section{P5.037 EXPERIENCE OF RAPID HIV TESTING INCREASES ITS ACCEPTABILITY TO CLINICAL STAFF IN PUBLIC SEXUAL HEALTH CLINICS IN SYDNEY}

doi:10.1136/sextrans-2013-051184.1081

'D P Conway, ${ }^{1} \mathrm{R}$ Guy, ${ }^{2,3} \mathrm{~A}$ McNulty, ${ }^{4,5} \mathrm{D}$ L Couldwell, ${ }^{6} \mathrm{~S}$ C Davies, ${ }^{7,3} \mathrm{D}$ E Smith, ${ }^{1} \mathrm{P}$

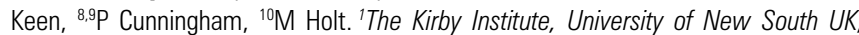

